\title{
The somato-dendritic A-type $\mathrm{K}^{+}$channel complex: a ménage à trois
}

\author{
Bernard Attali* \\ Department of Physiology and Pharmacology, Sackler Faculty of medicine, Tel Aviv University, Tel Aviv, Israel \\ *Correspondence: battali@post.tau.ac.il
}

\section{A commentary on}

DPP6 localization in brain supports function as a Kv4 channel associated protein

by Brian D. Clark, Elaine Kwon, Jon Maffie, Hyo-Young Jeong, Marcela Nadal, Pavel Strop and Bernardo Rudy

The somato-dendritic subthreshold-operating A-type $\mathrm{K}^{+}$current $\left(I_{S A}\right)$ plays a crucial role in neuronal excitability. It regulates spike timing and frequency, dampens the back propagation of action potentials into dendrites and has an important role in dendritic signal processing (Hoffman et al., 1997). Hence, any alteration of $I_{S A}$ current density or gating kinetics could then have important consequences on dendritic integration and plasticity.

$\mathrm{Kv} 4$ channel protein complexes represent the molecular correlate of the $I_{S A}$ current (Birnbaum et al., 2004; Maffie and Rudy, 2008). Studies in heterologous expression systems have shown that Kv4 pore-forming $\alpha$ subunits interact with two different auxiliary subunits: Kv-channel-interacting proteins (KChIPs) and the dipeptidylpeptidase-like proteins DPP6 and DPP10 (DPPX). Both auxiliary subunits affect channel gating and trafficking and their co-expression with $\mathrm{Kv} 4 \alpha$ subunits recapitulates the kinetics and gating properties of native $I_{S A}$.

The discovery of Nadal et al. (2003) showing that DPP6 proteins can be copurified with $\mathrm{Kv} 4.2 \mathrm{~K}^{+}$channel proteins from rat cerebellum and suggesting that they might function as auxiliary subunits of the channel complex, opened new interesting perspectives for the role of this structurally-related dipeptidyl aminopeptidase. Indeed, recent studies showed that mutations in the DPP6 gene are associated with human neurological diseases, including amyotrophic lateral sclerosis (ALS), autism and progressive spinal muscular atrophy. DDP6 is a type II transmembrane protein, highly expressed in the nervous system and belonging to the prolyl oligopeptidase family of serine proteases. It consists of a large extracellular C-terminal domain, a single membranespanning domain and a short alternatively spliced intracellular N-terminus. DPP6 has a dimeric structure and has lost a catalytic serine residue, suggesting that it is unlikely to function as a dipeptidyl aminopeptidase (Strop et al., 2004).

Though biochemical and functional data strongly suggest that DPP6 is an integral component of the $\mathrm{Kv} 4.2 \mathrm{~K}^{+}$channel complex and functions as an auxiliary subunit, the lack of good quality antibodies prevented high-resolution localization studies in native brain tissue. In the recent issue of Frontiers, Clark et al. (2008) provide a strong argument supporting the view that DPP6 is an integral part of native Kv4.2 channel complexes $\left(I_{S A}\right)$ in the brain. They raised antibodies against the whole extracellular domain of the protein, which was recombinantly expressed in baculovirus-infected Sf9 cells. Using this specific tool, they investigated DPP6 distribution in mouse brain and compared it with the distribution of $\mathrm{Kv} 4$ proteins (Clark et al., 2008). The three regions most prominently co-labeled with DPP6 and Kv4.2 antibodies were the striatum, the hippocampus and the cerebellar cortex. In the granule cells of the cerebellum, both Kv4.2 and DPP6 proteins were heavily stained in dendrites with a similar anterior-posterior gradient of expression. In line with the high density of $I_{S A}$ current found in dendrites of hippocampal pyramidal CA1 neurons (Hoffman et al., 1997), the dendrites of CA1-CA3 pyramidal cells were also prominently immunostained for both DPP6 and $\mathrm{Kv} 4.2$. In the molecular layer of the dentate gyrus, Kv4.2 and DPP6 staining co-localize in the dendrites of dentate granule cells. Thus, this study found remarkable simi- larities in the regional and cellular patterns of expression of Kv4.2 and DPP6 proteins. Similarities were also found in the dendritic subcellular pattern of expression of both proteins, notably in the CA1-CA3 hippocampal field and in the granule cells of the cerebellum.

Such findings strengthen the notion that DPP6 is an integral part of the Kv4 channel complex that underlies the somato-dendritic $I_{S A}$ current in neurons. However, this study further provides intriguing findings showing that one brain structure, the hippocampal mossy fibers, which contain the axons of the granule cells of the dentate gyrus, do not share this pattern of Kv4 and DPP6 co-expression. The hippocampal mossy fiber axons lack Kv4 proteins but are heavily labeled by DPP6 antibodies, particularly in the terminal fields in the stratum lucidum of the CA3 region, thereby suggesting that DPP6 proteins may have additional, Kv4unrelated functions.

Altogether, these recent studies on DPP6(X) proteins raise important issues. Most likely, the Kv4 channel complex contains two dimers of DPP6 proteins. If so, does DPP6 assemble as homodimer or heterodimer and how the KChIP subunits are engaged into the Kv4 channel complex? Are KChIP and DPP6 exerting their effects on $\mathrm{Kv} 4$ channel surface expression via different mechanisms? The absence of Kv4 $\alpha$ subunits and the strong expression of DPP6 in the hippocampal mossy fibers indicate that DPP6(X) proteins may have presynaptic functions. It will be important to investigate the possible role of DPP6 in transmitter release as well as in motor neuron function and excitotoxicity, regarded as one of the main processes involved in the pathogenesis of ALS and progressive muscular spinal atrophy. Addressing these challenging issues will highlight the importance of DPP6 in Kv4-related and - unrelated functions. 


\section{REFERENCES}

Birnbaum, S. G., Varga, A. W., Yuan, L. L., Anderson, A. E. Sweatt, J. D., and Schrader, L.A. (2004). Structure and function of Kv4-family transient potassium channels. Physiol. Rev. 84, 803-833.

Clark, B. D., Kwon, E., Maffie, J., Jeong, H. Y., Nadal, M., Strop, P., and Rudy, B. (2008). DPP6 localization in brain supports function as a $\mathrm{Kv} 4$ channel associated protein. Front. Mol. Neurosci. 1, 8 .

Hoffman, D. A., Magee, J. C., Colbert, C. M., and Johnston, D. (1997). $\mathrm{K}^{+}$channel regulation of signal propagation in dendrites of hippocampal pyramidal neurons. Nature 387, 869-875.
Maffie, J., and Rudy, B. (2008). Weighing the evidence for a ternary protein complex mediating A-type $\mathrm{K}^{+}$currents in neurons. J. Physiol. 586, 5609-5623.

Nadal, M. S., Ozaita, A., Amarillo, Y., Vega-Saenz de Miera, E., Ma, Y., Mo, W., Goldberg, E. M., Misumi, Y., Ikehara, Y., Neubert, T. A., and Rudy, B. (2003). The CD26-related dipeptidyl aminopeptidase-like protein DPPX is a critical component of neuronal A-type $\mathrm{K}^{+}$channels. Neuron 37, 449-461.

Strop, P., Bankovich, A. J., Hansen, K. C., Garcia, K. C., and Brunger, A. T. (2004). Structure of a human
A-type potassium channel interacting protein DPPX, a member of the dipeptidyl aminopeptidase family. J. Mol. Biol. 343, 1055-1065.

Received: 30 April 2009; published: 15 September 2009. Citation: Front. Neurosci. (2009) 3, 2: 158-159. doi: 10.3389/neuro.01.017.2009

Copyright (c) 2009 Attali. This is an open-access publication subject to an exclusive license agreement between the authors and the Frontiers Research Foundation, which permits unrestricted use, distribution, and reproduction in any medium, provided the original authors and source are credited.

\section{Details of the construction of perception: a closer look at illusory contours}

\author{
Johan Eriksson* and Lars Nyberg** \\ Physiology Section, Department of Integrative Medical Biology, Umeå University, Umeå, Sweden \\ *Correspondence: *johan.eriksson@physiol.umu.se; **lars.nyberg@physiol.umu.se
}

\section{A commentary on}

Retinotopic activation in response to subjective contours in primary visual cortex

by Marianne Maertens, Stefan Pollmann, Michael Hanke, Toralf Mildner and Harald Möller

Are we aware of neural activity in primary visual cortex? This question was asked in a classic paper written by Nobel laureate Francis Crick and Christof Koch more than a decade ago (Crick and Koch, 1995). By now, several studies have addressed the question, but so far consensus has not been reached (Rees, 2007; Tong, 2003). A recent study by Maertens et al. (2008) presents new relevant data on this issue. They demonstrated that activity in primary visual cortex (V1) is correlated with the perception of subjective contours. Maertens et al. used Kanizsa figures to induce a percept of illusory contours (IC, Figure 1), which are subjective in the sense that there is no actual change in luminance at the location of the perceived contour.

The (illusory) perception of the contours is dependent on coherent orientation of the inducers. Hence, by either aligning or misaligning the inducers, very similar

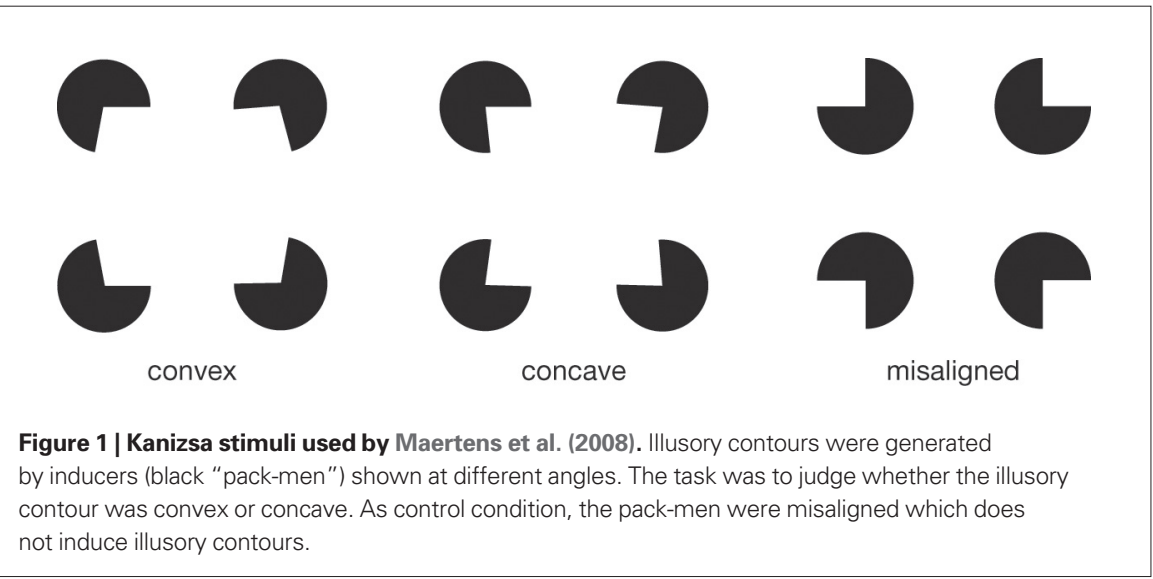

(local) physical parameters can create two subjectively different visual experiences. IC can therefore be used to provide information on neural correlates of conscious perception.

An important feature of Maertens et al.'s experiment was the localization of neural activity specific for contour perception. This was accomplished by using spatially specific localizer stimuli for the contours and inducers separately, as well as by using a spatially more precise imaging sequence than traditionally used ones. This enabled the conclusion that the IC were related to retinotopically specific regions within $\mathrm{V} 1$, thereby extending previous research on IC where IC-related activity at such a level of detail was not observed. The results also add to existing evidence regarding V1 involvement in conscious perception. In previous research on conscious perception, some but not other studies have observed correlated activity in V1. A factor that could account for the inconsistent results is the specific task requirements. Specifically, it has been suggested that there is a reverse hierarchical sequence for conscious perception such that global, holistic features are perceived first and the details are added if required (Hochstein and Ahissar, 2002). In the Maertens et al. paper, the angle of the 